\section{SAT0229 NUMBER NEEDED TO TREAT (NNT): IMPLICATION IN RHEUMATOLOGY CLINICAL PRACTICE}

${ }^{1} \mathrm{M}$ Osiri, ${ }^{2} \mathrm{ME}$ Suarez-Almazor, ${ }^{3} \mathrm{GA}$ Wells, ${ }^{3} \mathrm{P}$ Tugwell. 'Department of Medicine, Chulalongkorn University Hospital, Bangkok, Thailand; ${ }^{2}$ Health Services Research, Baylor College of Medicine, Houston, USA; ${ }^{3}$ Department of Medicine, University of Ottawa, Ottawa, Canada

\subsection{6/annrheumdis-2001.865}

Background A major challenge for rheumatologists is to translate the research data into treatment decision for their patients in clinical practice. One approach that is becoming increasingly used is the ?number needed to treat? (NNT), the reciprocal of absolute risk reduction, which is the number of patients who must be treated in order to obtain the benefit of interest in one new patient.

Objectives To calculate the NNT from rheumatology systematic reviews and clinical trials.

Methods We calculated the NNT from clinically important outcomes in rheumatology from the Cochrane Database of Systematic Reviews in the Cochrane Library Issue 2, 2000 or from the absolute risk differences of the primary outcomes from the original RCTs. If these outcomes were not available, the NNT was calculated from mean improvement of tender joint counts. Key words included musculoskeletal, rheumatology, and systematic reviews.

Results 15 systematic reviews and 11 original articles contributed the data for NNT calculation. The smallest NNT for the patients meeting the ACR20 criteria was from etanercept treatment compared to placebo (NNT 1.8;95\% CI 1.1,4.7). The NNT and 95\% CI for the patients treated with MTX, SSZ and leflunomide was $5.0(3.3,11), 3.6(2.5,6.7)$, and $3.9(2.7,6.6)$, respectively. The NNT for combination treatment (cyclosporin + MTX, infliximab + MTX, and prednisolone + MTX + SSZ) ranged from 2.6 to 4.8 . The NNT for azathioprine $(2.4 ; 95 \%$ CI $1.8,8.9)$ and high dose D-penicillamine $(5.2 ; 95 \%$ CI $2.8,34.2$ ) was calculated from mean change of the number of tender joints. The NNT for meeting the Paulus criteria in antimalarial-treated patients was $4.6(95 \%$ CI 2.5,25.6) and in injectable gold group was 4.0 (2.3,14.3). Among different NSAIDs used in hip OA, only etodolac (NNT 4.4; 95\% CI 2.4,24.4) and tenoxicam (NNT 3.8, 95\% CI 2.5,7.3) showed a significant NNT and 95\% CI for relieving pain over placebo.

Conclusion The NNT is a term translated from the less understandable research data to help clinicians in routine practice decision-making. Comparisons of the NNTs can be done among different treatment options for the same disease and outcome. The NNT should be adjusted for the baseline risk and treatment duration in individual patients.

\section{SAT0230 ECONOMIC ANALYSIS OF ROFECOXIB VERSUS NSAIDS: COMPARISON ACROSS DIFFERENT PROVINCES IN CANADA}

${ }^{1} \mathrm{MA}$ Marentette, ${ }^{2} \mathrm{C}$ Attard, ${ }^{3} \mathrm{JM}$ Pellissier, ${ }^{4} \mathrm{SX}$ Kong, ${ }^{1} \mathrm{C}$ Sauvageau. ${ }^{1}$ Health Economics, Patient Health, Merck Frosst Canada \& Co., Kirkland, QC, Canada; Innovus Research, Inc., Burlington, ON, Canada; ${ }^{3}$ Merck Research Laboratories, Blue Bell, PA, USA; ${ }^{4}$ Merck \& Co., Whitehouse Station, NJ, USA

\subsection{6/annrheumdis-2001.866}

\section{Background}

Objectives Rofecoxib (VIOXX) patients were shown in Phase III clinical trials to have significantly fewer clinically significant gastrointestinal (GI) adverse events than those who received non-selective NSAIDs for the treatment of osteoarthritis (OA). We evaluated the clinical and economic consequences of rofecoxib prescription in each of the ten Canadian provinces for patients over 65 who were assumed to have failed acetaminophen therapy.

Methods A cost-effectiveness analysis was conducted comparing rofecoxib to non-selective NSAIDs with respect to their gastrointestinal effects on patients with OA. A decision analytic model, with a time horizon of one year, was constructed focussing on the event rates from a pooled analysis of $8 \mathrm{Phase} \mathrm{IIb} / \mathrm{III}$ clinical trials. The number of perforations, ulcers and bleeds (PUBs) in each strategy was used as the primary measure of effectiveness. Province-specific inputs were incorporated to determine the relative impact of provincial differences in reimbursement policies, medication mix, prescription length, and dispensing fees on the economic results.

Results In six of the ten Canadian provinces, the replacement of traditional NSAIDs with rofecoxib was associated with fewer PUBs, but at a slightly higher annual cost per patient to the provincial governments (range: \$21.37 - \$133.42). In Alberta, Manitoba, Quebec and Newfoundland the use of rofecoxib was associated with an annual cost saving in addition to the clinical benefit of fewer PUBs. The variation in the model results across provinces was primarily due to the variation in average NSAID and GPA costs across the provinces. As drug choices differ between the provinces, the average daily cost of an NSAID was seen to vary between $\$ 0.49$ in Nova Scotia to $\$ 1.24$ in Newfoundland. Likewise, the average daily cost of a GPA ranged from \$0.72 in New Brunswick to \$2.11 in Alberta.

Conclusion Rofecoxib may represent a cost-effective alternative to non-selective NSAIDs for the treatment of OA in Canada, despite provincial differences in reimbursement policies and medication mix which generate varying burdens on payers and patients.

\section{SAT0231 HINDRANCES IN EFFECTING INSURANCES AMONG DUTCH ANKYLOSING SPONDYLITIS (AS) PATIENTS}

A Van Tubergen, A Boonen, S Van der Linden. Rheumatology, University Hospital Maastricht, Maastricht, The Netherlands

\subsection{6/annrheumdis-2001.867}

\section{Background}

Objectives To evaluate whether AS patients encounter hindrances in effecting different types of insurances after the diagnosis of AS.

Methods In January 2000, a questionnaire was sent to 129 Dutch AS patients inquiring about three different types of insurances: health insurance, additional work-disability insurance (for non-covered loss of income due to illness) and life insurance. We identified patients who had effected one or more insurances after the diagnosis of AS, and had encountered hindrances. Hindrances were defined as obligation to supply medical information, obligation to undergo medical examination, or restrictions in the insurance contract, such as higher premium, no restitution for AS-related problems etc. As a control group served those patients who also effected the above insurances, but before the diagnosis of AS.

Results After diagnosis of AS 15 out of 129 patients had effected a health insurance. Four of them were obliged to supply medical information versus 0 from the control group $(n=114)(p<$ 